\title{
Le texte dans l'exposition, un dispositif de tension permanente entre contrainte et créativité
}

Text' in an exhibition context - a matter of permanent tension between restrictions and creativity

\section{Marie-Sylvie Poli}

\section{(2) OpenEdition}

Journals

Édition électronique

URL : http://journals.openedition.org/ocim/377

DOI : $10.4000 /$ ocim. 377

ISSN : 2108-646X

Éditeur

OCIM

Édition imprimée

Date de publication : 1 novembre 2010

Pagination : 8-13

ISSN : 0994-1908

Référence électronique

Marie-Sylvie Poli, « Le texte dans l'exposition, un dispositif de tension permanente entre contrainte et créativité », La Lettre de l'OCIM [En ligne], 132 | 2010, mis en ligne le 01 novembre 2012, consulté le 19 avril 2019. URL : http://journals.openedition.org/ocim/377 ; DOI : 10.4000/ocim.377

Ce document a été généré automatiquement le 19 avril 2019

Tous droits réservés 


\title{
Le texte dans l'exposition, un dispositif de tension permanente entre contrainte et créativité
}

\author{
Text' in an exhibition context - a matter of permanent tension between \\ restrictions and creativity
}

Marie-Sylvie Poli

1 Avant de laisser la parole aux autres auteurs de ce numéro qui aborderont les questions des écrits pour les visiteurs non-voyants, de la traduction des textes dans l'exposition, des dispositifs de textes pour les publics enfants et de l'évaluation des écrits au musée, nous proposons de mettre l'accent sur les effets particuliers (autres que sémantiques) que les textes instaurent entre l'exposition et les visiteurs. Nous opterons donc pour une approche pragmatique (ou communicationnelle) du texte dans l'exposition et nous centrerons notre contribution sur une analyse de ce que le texte écrit «fait » à l'exposition, pour reprendre la célèbre formule du philosophe du langage John L. Austin (1911-1960) Quand dire, c'est faire. Faire, oui, mais quoi ? Et comment? Pour répondre à ce double questionnement, nous avancerons trois hypothèses : le texte parle l'exposition ; le texte écrit l'exposition; le texte représente l'exposition.

\section{Le texte parle des modèles de référence de l'exposition}

2 Parce qu'elle fonctionne comme un dispositif sémantique et communicationnel, toute exposition traite d'un thème principal et de sous thèmes qui s'y rattachent. Par ailleurs, on sait que toute exposition peut être envisagée comme une archive, à savoir comme le prolongement, l'interprétant, d'un ensemble de savoirs et de points de vue qui circulent dans l'espace des connaissances et des questionnements d'une société à un moment donné. L'exposition se constitue donc à partir d'un ensemble motivé d'opérations de sélections et de choix de discours qui portent : sur les sujets à aborder, sur les cadres de 
références retenus pour les traiter et sur les modes opératoires produits pour les exposer. Tous ces choix vont se retrouver exprimés, de manière plus ou moins explicite, dans le texte écrit de l'exposition. Autrement dit, le texte expose à sa manière les partis pris scénographiques et épistémiques des concepteurs de l'exposition.

3 Par ses caractéristiques stylistiques et linguistiques, le texte (depuis le titre jusqu'aux notices d'identification des objets) révèle les « grands modèles d'exposition » auxquels les concepteurs de l'exposition ont décidé de se référer, ont choisi de se confronter. Ainsi donne-t-il à lire les matrices (les archétypes) des textes d'expositions passées à partir desquels les auteurs ont écrit leur propre texte. Dans un musée de sciences ou un musée de société par exemple, un texte d'exposition qui comprend quantité de termes savants, de longues phrases explicatives, de reformulations d'expressions spécialisées, de chiffres commentés, de schémas et de graphiques, traduit la volonté des concepteurs de se situer dans le registre des expositions pédagogiques. Par opposition, une exposition au titre énigmatique, équipée d'un texte minimaliste, exprime la décision du commissaire d'exposition ("auteur») de se rapprocher de la catégorie des expositions d'art contemporain généralement fort peu équipées en dispositifs de médiation pour des publics qui connaissent peu ou mal les codes et les valeurs esthétiques de l'art contemporain. Dans ces deux cas extrêmes, on le comprend, le discours expographique n'est pas centré sur le visiteur (lecteur potentiel) mais sur le média exposition. Le texte n'est donc pas considéré comme susceptible d'instaurer une relation d'équité avec le visiteur.

4 Le texte étant par nature dialogique, c'est-à-dire à la fois nourri de textes antérieurs, et écrit pour faire réagir le lecteur, toutes les citations conscientes ou inconscientes sur lesquelles il s'appuie, toutes les références explicites ou implicites dont il est le porteparole agissent d'au moins deux façons sur l'exposition:

- elles disent les courants de pensée que l'exposition approuve ou ceux auxquels elle s'oppose ;

- elles trahissent les catégories socioculturelles des visiteurs auxquelles les concepteurs s'adressent en priorité.

Comme tout texte, le texte d'exposition ne dit pas tout. Il ne peut pas tout dire. Il ne veut pas tout dire. Ces non dits parlent eux aussi de ce que les auteurs n'ont pas voulu, pas pu aborder. Si l'on y prend garde, ce que ne manquent jamais de faire les experts scientifiques du domaine traité par une exposition thématique, ces silences disent bien des choses sur les limites scientifiques, éthiques ou politiques au-delà desquelles les commissaires ne veulent/ne peuvent aller. Soit parce qu'ils le décident, soit parce que leurs institutions de tutelle y verraient ombrage, jusqu'à les pénaliser parfois.

6 Dans l'exposition, le texte est toujours appliqué sur, tramé avec, inscrit, dans la matérialité des supports. Support papier, support mural, support numérique, support objet de collection ou support document fac-similé, ou supports récupérés lors d'une exposition itinérante. Tous ces choix de support du texte traduisent les partis pris de style de l'exposition et ce, de façon concrète. Ces choix trahissent tout d'abord le budget (de très confortable à dérisoire) dont a bénéficié l'exposition, ce qui la situe dans une échelle de valeurs à la fois économique (à quelle hauteur les partenaires institutionnels et privés ont financé) et symbolique (exposition événement national voire international, versus « petite » exposition). 
7 Les choix de supports disent aussi comment les concepteurs désirent connoter l'exposition, lui donner une image plus ou moins «high tech». En décidant d'utiliser surtout des outils de communication technologiques nouveaux et sophistiqués, ils disent leur envie que l'exposition apparaisse " dans le coup », moderne, dans l'air du temps. Ils affichent aussi leur goût pour ces supports technologiques, l'intérêt muséographique et communicationnel qu'ils y trouvent, particulièrement en termes de médiation.

En faisant le choix de supports dits traditionnels comme supports de texte de l'exposition (panneaux, textes écrits sur les cimaises, textes sur tissus servant de cloisons thématiques, cartels sur papier), les concepteurs ne trahissent pas seulement l'état de leur budget. Ils confirment leur attachement à une certaine tradition livresque de la mise en forme du texte; ils se reposent sur un dispositif visuel qui ne tombe pas en panne et ne nécessite pas de maintenance particulière; ils choisissent enfin de se rattacher à un mode d'expression de l'information et de la connaissance qui tient les médias audiovisuels ou numériques à l'écart. En choisissant aujourd'hui, compte tenu de leurs coûts modestes, de ne pas insérer d'écrans, de bornes interactives, de textes défilants ou de textes électroniques, les concepteurs situent symboliquement l'exposition hors du champ des médias technologiques de masse comme les téléphones mobiles, la télévision, les communications urbaines informatiques ou Internet.

9 Répétons-le encore une fois : la signification que le lecteur attribue au texte qu'il parcourt est toujours influencée par le support sur lequel le texte (sa forme et son contenu) parvient visuellement jusqu'à lui. Lors de la réception d'un écrit, le support sur lequel est placé cet écrit influence la signification que le lecteur attribue au matériau langagier (contraste visuel, symbolique des couleurs, symbolique des matériaux, représentations de modernité liées au support numérique versus représentations de rusticité liées au support bois par exemple).

\section{Le texte écrit le ton et pose le cadre de l'exposition}

Considérons maintenant la perspective pragmatique à partir de laquelle, premièrement tout texte n'a de sens qu'en contexte, qu'en situation de communication, et deuxièmement, tout texte influence la situation de communication où il se trouve.

D'une part on considère que tout texte vise un but explicite ou non, c'est-à-dire qu'il déploie une stratégie communicationnelle plus ou moins maîtrisée; d'autre part on postule que tout texte agit sur des croyances ou des comportements, c'est-à-dire qu'il se place sur le registre des compétences interprétatives de ses récepteurs potentiels.

12 Cela signifie que les desseins psychologiques des auteurs visant à toucher intellectuellement et émotionnellement le visiteur/lecteur se traduisent toujours aussi par des choix langagiers. Ces choix de typographie, de mots, de phrasé, de ponctuation, de mise en page, sont le résultat des multiples opérations de sélection de langue opérées par les auteurs.

13 Même si l'on sait fort bien que dans l'exposition - comme dans toute autre situation de communication à dimension langagière - le sens n'est jamais imposé par le concepteur du médium, mais résulte toujours de négociations entre les interprétations du récepteur et les mots du concepteur, on voit bien tout l'intérêt que les « expographes » c'est-à-dire les concepteurs d'expositions peuvent trouver à travailler le texte très en amont du montage de l'exposition, dès que se pose la question du « genre d'exposition » qu'ils veulent créer. 
Ou pour le dire autrement, il est intéressant, au moment même de l'élaboration du projet d'exposition, de travailler le texte comme un outil stratégique de communication capable d'agir sur le discours de l'exposition dans sa globalité, à partir des processus pragmatiques (ou ressources) qui le caractérisent.

Ces ressources sont d'au moins quatre ordres : le ton du texte d'exposition (savant, drôle, pédagogique, polémique, poétique...); le format du texte d'exposition (livresque, journalistique, encyclopédique, événementiel...) ; la syntaxe du texte d'exposition (mise en intrigue, organisation en séquences du niveau macro au niveau micro, correspondance entre les énoncés et les objets); le lexique du texte d'exposition (quelles terminologies, quels univers de sens, quels temps, quels modes, quels outils grammaticaux, quelle ponctuation...).

Écrire du texte d'exposition peut donc être envisagé dans cette optique, comme opérer des choix qui relèvent de ces quatre types de ressources à la fois: le ton, le format, la syntaxe, le lexique.

En accentuant le trait, on pourrait dire que :

- le recours très fréquent à des verbes au conditionnel, à des adverbes de doute et à des formes interrogatives participera en partie à donner un ton dialectique ou polémique au texte d'exposition et donc à l'exposition elle-même ;

- le fait de travailler volontairement le registre poétique de la langue dans la plupart des énoncés donnera une coloration poétique au texte d'exposition et donc à l'exposition ellemême ;

- le fait de choisir, dès avant la conception de l'exposition, que tous les textes seront dits pour être entendus par des non-voyants, va d'une part forcer à réduire la masse des textes exposés, d'autre part imposer d'inventer une langue idoine et poétique qui cherche à dire ce qui n'est pas vu ou très peu vu ou partiellement vu ;

- décider par avance de distiller des jeux de mots ou des boutades dans les notices et les cartels d'une exposition crée un rapport volontairement distancié et humoristique. Cela nous effraie encore en France où l'on pense toujours que les visiteurs d'une exposition seraient gênés par ce type d'initiative. Les succès de certaines expositions ayant relevé le défi sont pourtant la preuve de la pertinence de cette écriture ;

- donner la parole à d'autres locuteurs que les commissaires d'exposition (artistes, écrivains, chercheurs, anonymes) et écrire dès le début du projet le texte de l'exposition comme des conversations entre deux ou plusieurs personnages (avec leurs «langues») induit une exposition qui bruisse, une exposition conversationnelle (idée du débat) et polyphonique (à plusieurs voix);

- donner la préférence à un texte composé d'une arborescence de parties, sous-parties, notices, cartels qui proposent des savoirs savants, des débats de chiffres, des définitions recherchées, c'est opter ainsi pour un format encyclopédique et une syntaxe hypertextuelle du texte de l'exposition. Ce cadre implique en retour une scénographie qui privilégie la lecture du discours verbal par rapport aux autres accès à l'émotion et à la connaissance.

\section{La confiance dans les contenus très rarement remise en question}

Il peut paraitre trivial de rappeler pour la énième fois ce que tous les professionnels de l'exposition savent certainement déjà, mais que trop peu encore mettent en pratique. 

lui donner envie de lire; des textes qui comportent plus de deux cents mots ne sont lus qu'en partie, voire pas lus du tout ; les chiffres, schémas, tableaux, autres écritures non verbales dès qu'ils sont fréquents et denses sont contre productifs en terme de plaisir à la visite pour la grande majorité des publics; les écrits d'écran donnant accès à des hypertextes multimodaux (écrit, image, son) pourtant utilisés par la plupart des publics dans la sphère privée ou professionnelle, demandent une telle disponibilité en temps et en concentration cognitive qu'ils ne sont effectivement utilisés que par une infime partie des visiteurs ; les formules emphatiques et les cascades de références savantes mettent le visiteur en situation d'infériorité, de frustration, parfois de déni vis-à-vis de l'exposition ; les textes proposés sous forme d'aide à la visite ne sont efficients que s'ils se rapportent visiblement aux expôts scénographiés ; les visiteurs traduisent en indices signifiants les registres sémiotiques de formes et de couleurs; les textes destinés aux enfants sont aussi lus par les adultes et réciproquement; les textes poétiques sont d'excellents vecteurs de la connaissance...

Dans un autre registre que celui de l'efficacité cognitive stricto sensu des textes d'exposition, les études approfondies menées auprès des publics révèlent que la relation que chacun instaure avec le texte en dit long sur les représentations que le visiteur a de lui-même, de lui en tant que sujet social dans ses relations aux musées et à la culture (ou aux loisirs).

Ainsi, il est intéressant de rappeler que d'après les résultats de nos recherches dans les expositions de beaux-arts et dans les expositions de sciences, la plupart des visiteurs attribuent un tel crédit de confiance et d'expertise aux musées en France qu'il est plus rare de rencontrer des personnes qui disent que les textes sont mal écrits, que des personnes qui se disent pas assez compétentes pour comprendre ce qu'on a tenté de leur transmettre ${ }^{1}$.

21 Dans un autre ordre d'idée, on constate que les visiteurs experts dotés d'un fort capital culturel (les amateurs cultivés) estiment généralement qu'il y a trop de texte, trop d'explications, et qu'ils regrettent que le musée les considère comme des apprenants, voire des enfants. Une façon de dire qu'ils ne se sentent pas pris en considération en tant que visiteurs cultivés.

Parfois au contraire, certains visiteurs trouvent les textes en telle empathie avec leurs types d'attentes qu'ils parlent de leur lecture comme d'une relation de connivence et de confiance instaurée entre eux et les auteurs de l'exposition.

Le texte ne se limite pas, on le sait, à transmettre des informations. Le texte communique aussi des valeurs et des repères culturels, donnant parfois au visiteur l'occasion de se positionner par rapport aux idéaux et aux visions de l'institution qui montre l'exposition. Dans le meilleur des cas, le texte peut aussi conduire le visiteur à reconsidérer ses propres valeurs, ses propres croyances.

Pour évoquer la force du lien qui nous lie à nos choix en termes de lecture, un proverbe dit : « dis moi ce que tu lis et je te dirai qui tu es ». Transposé au monde des musées, on peut le paraphraser en : "dis moi quel texte d'exposition tu aimes et je te dirai quel visiteur tu es ». Car les recherches en muséologie sur la réception du texte dans l'exposition démontrent que pour les visiteurs, le texte participe fortement du rapport intime qu'ils entretiennent avec l'exposition, et qu'en retour l'exposition entretient avec eux. 


\section{Conclusion} mise en espace esthétique d'objets de patrimoine ou d'objets de témoignage; il ne s'agit pas pour autant des seuls décors ni des seules trouvailles technologiques des scénographes; il ne s'agit pas strictement de l'impact esthétique ou émotionnel des images, ni de la force didactique de concepts savants; il ne s'agit pas seulement non plus des seuls textes exprimant des points de vue et des savoirs. Il s'agit de tout cela à la fois, le texte participant à la médiation du sens de l'exposition comme un dispositif sémiotique et intellectuel de communication de premier plan. D'où notre postulat selon lequel le texte « écrit » l'exposition - c'est-à-dire fait de l'exposition un dispositif intellectuel dont la construction du sens est soumise aux cadres socioculturels et psycho cognitifs de chaque visiteur - au même titre que tous les autres vecteurs du discours d'exposition précédemment rappelés.

\section{NOTES}

1. Poli, M.-S. L'écrit au musée : comment les dispositifs de textes informatifs sont-ils perçus par les visiteurs? Cahier de Recherche de l'École doctorale en Linguistique française, université francoitalienne (Brescia, Milan, Turin, Trieste/Paris, Grenoble), n 1, 2008, pp. 15-31.

\section{RÉSUMÉS}

En s'intéressant à la dimension communicationnelle de l'écrit mis en texte dans l'exposition, les réflexions proposées ici s'attachent à montrer les effets matériels et symboliques du texte sur l'exposition envisagée comme média culturel.

By looking at the communication dimension of written texts in an exhibition, the following ideas put forward by the author try to indicate the material and symbolic effects of text on an exhibition when conceived as 'cultural media'.

\section{INDEX}

Mots-clés : exposition, médiation, texte 


\section{AUTEUR}

MARIE-SYLVIE POLI

Marie-Sylvie Poli est professeur à l'université Pierre Mendès France de Grenoble, équipe Culture et Communication, centre Norbert Élias, université d'Avignon et des Pays du Vaucluse marie-sylvie.poli@upmf-grenoble.fr 